\title{
Coupling Constant Unification in Extensions of Standard Model
}

\author{
Ling-Fong Li, and Feng Wu \\ Department of Physics, Carnegie Mellon University, Pittsburgh, PA 15213
}

May 28, 2018

\begin{abstract}
Unification of electromagnetic, weak, and strong coupling constants is studied in the extension of standard model with additional fermions and scalars. It is remarkable that this unification in the supersymmetric extension of standard model yields a value of Weinberg angle which agrees very well with experiments. We discuss the other possibilities which can also give same result.
\end{abstract}

One of the attractive features of the Grand Unified Theory is the convergence of the electromagnetic, weak and strong coupling constants at high energies and the prediction of the Weinberg angle [1, [3]. This lends a strong support to the supersymmetric extension of the Standard Model. This is because the Standard Model without the supersymmetry, the extrapolation of 3 coupling constants from the values measured at low energies to unification scale do not intercept at a single point while in the supersymmetric extension, the presence of additional particles, produces the convergence of coupling constants elegantly [4, or equivalently the prediction of the Weinberg angle agrees with the experimental measurement very well[5]. This has become one of the cornerstone for believing the supersymmetric Standard 
Model and the experimental search for the supersymmetry will be one of the main focus in the next round of new accelerators.

In this paper we will explore the general possibilities of getting coupling constants unification by adding extra particles to the Standard Model[2] to see how unique is the Supersymmetric Standard Model in this respect[?]. We will compute the contribution of various types of new particles to the evolution of the coupling constants and study their effects on the unification. These results will also be useful for checking models with extra particles to see whether they have a satisfactory unification of coupling constants.

\section{Evolution of Coupling Constants}

In this section we will set up the framework to discuss the unification in the $S U$ (5) type of model. Recall that the evolution of the coupling constants $g_{3}, g_{2}, g_{1}$ of the subgroups $S U(3)_{C}, S U(2)_{W}, U(1)_{Y}$ at one loop are given by

$$
\frac{1}{g_{i}^{2}(\mu)}=\frac{1}{g_{i}^{2}\left(\mu_{0}\right)}+2 b_{i} \ln \left(\frac{\mu}{\mu_{0}}\right), \quad i=1,2,3
$$

where $b_{i}$ are the coefficients in $\beta$-function of the renomalization group equations 6 ,

$$
\frac{d g_{i}}{d(\ln \mu)}=-b_{i} g_{i}^{3}
$$

Note that the coupling constants $g_{2}, g_{1}$ are related to the usual coupling constants, $g, g^{\prime}$ in $S U(2) \times U(1)$ by

$$
g=g_{2}, \quad g^{\prime}=\sqrt{\frac{3}{5}} g_{1}
$$

and Weinberg angle is given by

$$
\sin ^{2} \theta_{W}=\frac{g^{\prime 2}}{g^{2}+g^{\prime 2}}
$$


At the unification scale $M_{X}$, we have

$$
g_{1}\left(M_{X}\right)=g_{2}\left(M_{X}\right)=g_{3}\left(M_{X}\right)=g_{5}
$$

where $g_{5}$ is the gauge coupling of the $S U(5)$ group. Using the relation, $e=g \sin \theta_{W}$, we can write

$$
\begin{gathered}
\frac{1}{\alpha_{3}(\mu)}=\frac{1}{\alpha_{5}}+8 \pi b_{3} \ln \left(\frac{\mu}{M_{X}}\right) \\
\frac{1}{\alpha(\mu)} \sin ^{2} \theta_{W}=\frac{1}{\alpha_{5}}+8 \pi b_{2} \ln \left(\frac{\mu}{M_{X}}\right) \\
\frac{3}{5} \frac{1}{\alpha(\mu)} \cos ^{2} \theta_{W}=\frac{1}{\alpha_{5}}+8 \pi b_{1} \ln \left(\frac{\mu}{M_{X}}\right)
\end{gathered}
$$

where $\alpha=\frac{e^{2}}{4 \pi}, \alpha_{3}=\frac{g_{3}^{2}}{4 \pi}, \alpha_{5}=\frac{g_{5}^{2}}{4 \pi}$. Eliminating the unification scale $M_{X}$ from Eqs (68) we get

$$
\sin ^{2} \theta_{W}=\frac{1}{\left(1+\frac{8}{5} \bar{b}\right)}\left[\frac{3}{5} \bar{b}+\left(\frac{\alpha}{\alpha_{3}}\right)\right]
$$

where

$$
\bar{b}=\frac{b_{3}-b_{2}}{b_{2}-b_{1}}
$$

and all coupling constants $\alpha_{i}$ are evaluated at $\mu=M_{Z}$. For convenience, we can solve $\bar{b}$ in terms of $\sin ^{2} \theta_{W}$,

$$
\bar{b}=\frac{\left(\frac{\alpha}{\alpha_{3}}-\sin ^{2} \theta_{W}\right)}{\left(\frac{8}{5} \sin ^{2} \theta-\frac{3}{5}\right)}
$$


Using the experimental values, $\frac{\alpha}{\alpha_{3}}=.0674$ and $\sin ^{2} \theta_{W}=0.231$, [7] we get

$$
\bar{b}=0.71
$$

which is value needed in the unification to get the right value for $\sin ^{2} \theta_{W}$. Note that in the unification of the Standard Model without supersymmetry, we have $\bar{b}=\frac{1}{2}$ which gives $\sin ^{2} \theta_{W}=0.204$, and is significantly different from the experimental value. The inclusion of Higgs scalars will have a very small effect and will not change this result significantly. Or equivalently, if we use the experimental value for $\sin ^{2} \theta_{W}$, coupling constants, when the extrapolated to the unification scales, will not converge to a single coupling constant. Comparing the Standard Model value with Eq (12) we see that we need to increase $\bar{b}$ in the Standard Model to satisfy the experimental value.

Recall that various contributions to the $\beta$-function for the gauge coupling constants are of the form,

$$
\beta_{i}=\frac{-g_{i}^{3}}{16 \pi^{2}}\left[\frac{11}{3} t_{2}^{(i)}(V)-\frac{2}{3} t_{2}^{(i)}(F)-\frac{1}{3} t_{2}^{(i)}(S)\right]
$$

Here $t_{2}^{(i)}(V), t_{2}^{(i)}(F)$ and $t_{2}^{(i)}(S)$ are the contributions to $\beta_{i}$ from gauge bosons, Weyl fermions, and complex scalars respectively. For fermions in the representation with representation matrices $T^{a}$, they are given by

$$
t_{2}(F) \delta^{a b}=\operatorname{Tr}\left\{T^{a}(F) T^{b}(F)\right\}
$$

where the trace means summing over all members of the multiplets. Similarly, for the gauge bosons and scalar contributions, $t_{2}(V)$ and $t_{2}(S)$. For example, for the gauge bosons, $T^{a \prime} s$ are the matrices for the adjoint representations 
and for the gauge group $S U(n), t_{2}^{(n)}(V)$ is given by,

$$
\begin{array}{ll}
t_{2}^{(n)}(V)=n, & \text { for } n>2, \\
t_{2}^{(n)}(V)=0, & \text { for } n=1
\end{array}
$$

Note that $t_{2}^{(i)}(R), R=V, S, F$ are pure group theory factors and are independent of the spin of the particles. For the low rank representations in the $S U(n)$ group they are given by,

\begin{tabular}{|c|c|}
\hline & $t_{2}^{(n)}$ \\
\hline fundamental rep & $\frac{1}{2}$ \\
\hline adjoint rep & $n$ \\
\hline 2nd rank antisymmetric tensor & $\frac{n-2}{2}$ \\
\hline 2nd rank antisymmetric tensor & $\frac{n+2}{2}$ \\
\hline
\end{tabular}

Since $\frac{1}{16 \pi^{2}}$ is common to all $\beta$-functions and will be cancelled out in the ratio in $\bar{b}$ in Eq (10) we will neglect it in writing out the coefficient $b_{n}$,

$$
b_{n}=\left[\frac{11}{3} t_{2}^{(n)}(V)-\frac{2}{3} t_{2}^{(n)}(F)-\frac{1}{3} t_{2}^{(n)}(S)\right]
$$

For convenience, we can use the parameter $\bar{b}$ given in Eq (12) to discuss the contribution to the Weinberg angle $\theta_{W}$ from models with new particles.

$$
\bar{b}=\frac{b_{32}}{b_{21}}
$$

where

$$
b_{32} \equiv b_{3}-b_{2}, \quad b_{21} \equiv b_{2}-b_{1}
$$


We will separate the contributions into gauge bosons, fermions, and scalars,

$$
\begin{aligned}
& b_{32}=b_{32}(V)+b_{32}(F)+b_{32}(S) \\
& b_{21}=b_{21}(V)+b_{21}(F)+b_{21}(S)
\end{aligned}
$$

Note that for a complete multiplet of $S U(5)$, their contributions to $b_{32}$ and $b_{21}$ cancel out in the combination in Eq (16). This can be seen as follows. Let $T^{(a)}(R)$ be the matrices for some representation $R$ of $S U(5)$ group. Then the coefficient $t_{2}(R)$ of the second Casmir operator given by

$$
t_{2}(R) \delta^{a b}=\operatorname{tr}\left\{T^{(a)}(R) T^{(b)}(R)\right\}
$$

will be the same when we take $T^{(a)}(R) \in S U(3), S U(2)$ or $U(1)$,

$$
t_{2}^{(3)}(R)=t_{2}^{(2)}(R)=t_{2}^{(1)}(R)
$$

Thus their contributions will cancel out in the combination $b_{3}-b_{2}$ or $b_{2}-b_{1}$. In other words, a complete multiplet of $S U(5)$, e.g. $\mathbf{5}$ or $\mathbf{1 0}$, will not effect the prediction of the Weinberg angle, even though they will effect the evolution of each individual coupling constant. Here we assume that all members of the $S U$ (5) multiplets survive to low energies. This is the case for the fermion contributions in the Standard Model. Thus for the studies of the Weinberg angle, we need to consider only the incomplete $S U(5)$ multiplets, i.e. those $S U(3) \times S U(2) \times U(1)$ multiplets which do not combine into a complete $S U$ (5) multiplets. The reason that we can have the incomplete multiplets in the unification theory has to do with the decoupling of superheavy particles. For example, in the standard $S U(5)$ unification, only gauge bosons in subgroups $S U(3) \times S U(2) \times U(1)$ survive to low energies, while the other gauge bosons, e.g. leptoquarks, are superheavy and decouple. Similarly, only Higgs bosons in $S U(2)$ doublet will contribute to the $\beta$-function. 


\section{Contribution to $\theta_{W}$ from Various Multiplets}

The contributions of $S U(3) \times S U(2) \times U(1)$ gauge bosons are given by, using Eq (15)

$$
b_{3}(V)=11, \quad b_{2}(V)=\frac{22}{3}, \quad b_{1}(V)=0
$$

and they give

$$
b_{32}(V)=\frac{11}{3}, \quad b_{21}(V)=\frac{22}{3}
$$

The contribution from the Higgs scalars are

$$
b_{32}(S)=\frac{1}{6}, \quad b_{21}(S)=-\frac{1}{15}
$$

Since these contributions are very small, they are not included in the usual analysis.

We now consider the effects of various low rank multiplets to the Weinberg angle, or the parameter $\bar{b}$ in Eq (16). The results for the scalars and fermions are listed separately in the following tables:

(a) Scalars: 


\begin{tabular}{|c|c|c|c|c|c|c|}
\hline$S U(3)$ & $S U(2)$ & $b_{1}(s)$ & $b_{2}(s)$ & $b_{3}(s)$ & $b_{32}(S)$ & $b_{21}(S)$ \\
\hline color singlet & $\operatorname{doublet}\left(\begin{array}{c}\phi^{0} \\
\phi^{-}\end{array}\right)$, & $-\frac{1}{10}$ & $-\frac{1}{6}$ & 0 & $\frac{1}{6}$ & $-\frac{1}{15}$ \\
\hline "I & triplet $\left(\begin{array}{c}\phi^{+} \\
\phi^{0} \\
\phi^{-}\end{array}\right)$, & 0 & $-\frac{2}{3}$ & 0 & $\frac{2}{3}$ & $-\frac{2}{3}$ \\
\hline "1 & triplet $\left(\begin{array}{c}\phi^{++} \\
\phi^{+} \\
\phi^{0}\end{array}\right)$, & $-\frac{3}{5}$ & $-\frac{2}{3}$ & 0 & $\frac{2}{3}$ & $-\frac{1}{15}$ \\
\hline "1 & Singlet $\phi^{+}$, & $-\frac{1}{5}$ & 0 & 0 & 0 & $\frac{1}{5}$ \\
\hline$\prime \prime$ & Singlet $\phi^{++}$, & $-\frac{4}{5}$ & 0 & 0 & 0 & $\frac{4}{5}$ \\
\hline 11 & Singlet $\phi^{0}$ & 0 & 0 & 0 & 0 & 0 \\
\hline color triplet & doublet $\left(\begin{array}{l}\psi \\
d\end{array}\right)$ & $-\frac{1}{30}$ & $-\frac{1}{2}$ & $-\frac{1}{3}$ & $\frac{1}{6}$ & $-\frac{7}{15}$ \\
\hline "I & singlet $\psi$ & $-\frac{4}{15}$ & 0 & $-\frac{1}{6}$ & $-\frac{1}{6}$ & $\frac{4}{15}$ \\
\hline "I & Singlet $d$ & $-\frac{1}{15}$ & 0 & $-\frac{1}{6}$ & $-\frac{1}{6}$ & $\frac{1}{15}$ \\
\hline "I & 1 generation of squarks & $-\frac{11}{30}$ & $-\frac{1}{2}$ & $-\frac{2}{3}$ & $-\frac{1}{6}$ & $-\frac{2}{15}$ \\
\hline
\end{tabular}


(b) Fermions:

\begin{tabular}{|c|c|c|c|c|c|c|c|}
\hline$S U(3)$ & \multicolumn{2}{|c|}{$S U(2)$} & $b_{1}(f)$ & $b_{2}(f)$ & $b_{3}(f)$ & $b_{32}(f)$ & $b_{21}(f)$ \\
\hline color singlet & doublet & $\begin{array}{l}\nu \\
l^{-}\end{array}$ & $-\frac{1}{5}$ & $-\frac{1}{3}$ & 0 & $\frac{1}{3}$ & $-\frac{2}{15}$ \\
\hline " & triplet & $\begin{array}{l}l^{+} \\
l^{0}\end{array}$ & 0 & $-\frac{4}{3}$ & 0 & $\frac{4}{3}$ & $-\frac{4}{3}$ \\
\hline " & triplet & $\left.\begin{array}{l}l^{++} \\
l^{+} \\
l^{0}\end{array}\right)$ & $-\frac{6}{5}$ & $-\frac{4}{3}$ & 0 & $\frac{4}{3}$ & $-\frac{2}{15}$ \\
\hline " & singlet & $l^{+}$ & $-\frac{2}{5}$ & 0 & 0 & 0 & $\frac{2}{5}$ \\
\hline " & singlet & $l^{0}$ & 0 & 0 & 0 & 0 & 0 \\
\hline color triplet & doublet & $\left.\begin{array}{l}u \\
d\end{array}\right)$ & $-\frac{1}{15}$ & -1 & $-\frac{2}{3}$ & $\frac{1}{3}$ & $-\frac{14}{15}$ \\
\hline " & singlet & $u_{R}$ & $-\frac{8}{15}$ & 0 & $-\frac{1}{3}$ & $-\frac{1}{3}$ & $\frac{8}{15}$ \\
\hline " & singlet & $d_{R}$ & $-\frac{2}{15}$ & 0 & $-\frac{1}{3}$ & $-\frac{1}{3}$ & $\frac{2}{15}$ \\
\hline " & 1 generation & of quarks & $-\frac{11}{15}$ & -1 & $-\frac{4}{3}$ & $-\frac{1}{3}$ & $-\frac{4}{15}$ \\
\hline color octet & glun & & 0 & 0 & -2 & -2 & 0 \\
\hline
\end{tabular}

Remarks :As it is evident in Eq (13) with same $S U(3) \times S U(2) \times U(1)$ quantum numbers the contribution from the scalars are $\frac{1}{2}$ of the fermions contributions. 


\section{Supersymmetric extension of standard model and generalization}

As we have discussed before, we need to consider only the incomplete multiplets. The particles needed to be included are given below,

1. Scalars:

2 Higgs doublets $\quad H=\left(\begin{array}{c}H^{+} \\ H^{0}\end{array}\right) \quad b_{32}(H)=\frac{1}{3}, \quad b_{21}(H)=-\frac{2}{15}$

2. Fermions

$$
\begin{array}{crrr}
\text { glunios } & \tilde{g}^{a}, a=1, \ldots 8 & b_{32}\left(\tilde{g}^{a}\right)=-2 & b_{21}\left(\tilde{g}^{a}\right)=0 \\
\text { Winos } & \tilde{W}=\left(\begin{array}{c}
\tilde{W}^{+} \\
\tilde{W}^{0} \\
\tilde{W}^{-}
\end{array}\right) & b_{32}(\tilde{W})=\frac{4}{3} & b_{21}(\tilde{W})=-\frac{4}{3} \\
\text { binos } & \tilde{b} & b_{32}(\tilde{b})=0 & b_{21}(\tilde{b})=0 \\
2 \text { Higginos } & \tilde{h}=\left(\begin{array}{c}
\tilde{h}^{0} \\
\tilde{h}^{-}
\end{array}\right) & b_{32}(\tilde{h})=\frac{2}{3} & b_{21}(\tilde{h})=-\frac{4}{15}
\end{array}
$$

Thus the total contributions are

$$
b_{32}=\frac{1}{3}, \quad b_{21}=-\frac{26}{15}
$$

and using Eq (16), we get for $\bar{b}$,

$$
\bar{b}=\frac{\frac{11}{3}+\frac{1}{3}}{\frac{22}{3}-\frac{26}{15}}=.715
$$

This agrees with the experimental measurement very well and gives a strong support for the supersymmetric extension of Standard Model. Note that the 
result $b_{32}=\frac{1}{3}$ comes from a cancellation between large glunios contribution and that of winos and Higginos. Thus the mechanism to produce the right value for the Weinberg angle in the supersymmetric extension of Standard Model is quite complicate. This makes the SSM somewhat unique in the sense the extra particles which give all these cancellations are not arbitrary but are required by the principle of supersymmetry.

We will now discuss the other examples which can give satisfactory answer for the Weinberg angle. Since the supersymmetric extension of Standard Model has been so successful in the prediction of Weinberg angle, we will now give the possibilities of other multiplets which give the same contribution. We will use a simpler notation for the multiplet structure, $(\mathbf{a}, \mathbf{b})_{Y}^{f}$, denote a fermion multiplet transforms as representations $\mathbf{a}, \mathbf{b}$ under $S U(3) \times S U(2)$ with hypercharge $Y$. Similarly, $(\mathbf{a}, \mathbf{b})_{Y}^{S}$ for the scalar multiplets. To present the result, we group together the multiplets with same contributions for the coefficients functions, $b_{3}, b_{2}$, and $b_{1}$.

1. $b_{32}=-2, b_{21}=0$ (glunio-like states)

\begin{tabular}{|c|c|c|c|}
\hline$(\mathbf{8}, \mathbf{1})_{0}^{f}$ & $(\mathbf{6}, \mathbf{1})_{0}^{f} \oplus(\overline{\mathbf{3}}, \mathbf{1})_{0}^{f}$ & $(\mathbf{3}, \mathbf{1})_{0}^{f} \times 6$ & $(\mathbf{8}, \mathbf{1})_{0}^{s} \times 2$ \\
\hline$(\mathbf{8}, \mathbf{1})_{0}^{s} \oplus(\mathbf{6}, \mathbf{1})_{0}^{s}(\mathbf{3}, \mathbf{1})_{0}^{s}$ & $(\mathbf{6}, \mathbf{1})_{0}^{s} \times 2 \oplus(\overline{\mathbf{3}}, \mathbf{1})_{0}^{f}$ & $(\mathbf{6}, \mathbf{1})_{0}^{s} \oplus(\overline{\mathbf{3}}, \mathbf{1})_{0}^{f} \times 2$ & \\
\hline
\end{tabular}

2. $b_{32}=\frac{4}{3}, b_{21}=-\frac{4}{3}$ (Wino-like states)

\begin{tabular}{|l|l|l|}
\hline$(\mathbf{1}, \mathbf{3})_{0}^{f}$ & $(\mathbf{1}, \mathbf{3})_{0}^{s} \times 2$ & $(\mathbf{1}, \mathbf{2})_{0}^{s} \times 8$ \\
\hline
\end{tabular}

3. $b_{32}=\frac{2}{3}, b_{21}=-\frac{4}{15}$ (Higgio-like states)

\begin{tabular}{|c|c|c|}
\hline$(\mathbf{1}, \mathbf{2})_{-1}^{f} \times 2$ & $(\mathbf{1}, \mathbf{2})_{-1}^{s} \times 4$ & $(\mathbf{1}, \mathbf{3})_{0}^{s} \oplus(\mathbf{1}, \mathbf{1})_{2}^{f}$ \\
\hline$(\mathbf{1}, \mathbf{2})_{-1}^{f} \oplus(\mathbf{1}, \mathbf{2})_{-1}^{s} \times 2$ & $(\mathbf{1}, \mathbf{3})_{0}^{s} \oplus(\mathbf{1}, \mathbf{1})_{2}^{s} \times 2$ & \\
\hline
\end{tabular}


4. $b_{32}=\frac{1}{3}, b_{21}=-\frac{2}{15}$ (Higgs-like states)

$$
(\mathbf{1}, \mathbf{2})_{-1}^{s} \times 2
$$

Note that this last group includes the Standard Model Higgs contribution.

Clearly, if take one set of multiplet from each group, the total contribution will give the same Weinberg angle as the supersymmetry Standard Model. The list given above is constructed by mimicking those multiplets of the supersymmetry Standard Model and are by no means the only possibilities. We just want to demonstrate the existence of other examples. None of these possibilities, except for the supersymmetric one, are realized in any realist phenomenological model. For the case of new fermions multiplets, one might worry about the anomaly cancellation. However, as we have discussed before, only the incomplete multiplets are relevant for the Weinberg angle and it is possible to cancel the anomalies by fermion multiplets which decouple at very high energies.

\section{References}

[1] H. Georgi, S. L. Glashow, Phys. Rev. Lett. 32, 438, (1974).

[2] For earlier work in this direction see for example, Z. Berezhiani, I. Gogoladze and A. Kobakhidze, Phys.Lett.B522, 107, (2001).

[3] H. Georgi, H. Quinn, and S. Weinberg, Phys. Rev. Lett. 33, 451 (1974).

[4] S. Dimopoulos, S. Raby, and F. Wilczek, Phys. Rev. D24, 1681( 1981); M. Einhorn and D. Jones, Nucl. Phys. B196, 475 (1982);W. J. Marciano and G. Senjanovic, Phys.Rev.D25, 3092, (1982).

[5] U. Amaldi et. al. Phys. Rev. D36, 1385, (1987) 
[6] M. Gell-Mann, and F. E. Low, Phys. Rev. 95, 1300 (1954); C. G. Callan, Phys. Rev. D2, 1541 (1970); Symanzik, Comm. Math. Phys. 18, 227 (1970).

[7] Particle Data Group, Phys. Rev. D66, (2002) 